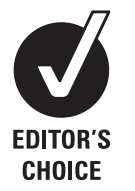

${ }^{1}$ Department of Ophthalmology, Kyoto Prefectural University of Medicine, Graduate School of Medicine, Kyoto, Japan ${ }^{2}$ Research Center for Inflammation and Regenerative Medicine, Doshisha University, Kyoto, Japan

\section{Correspondence to}

Takahiro Nakamura, Department of Ophthalmology, Kyoto Prefectural University of Medicine, Kawaramachi Hirokoji, Kamigyo-ku, Kyoto 602-0841 Japan; tnakamur@koto.kpu-m.ac.jp

TN and KT contributed equally to this work.

Accepted 21 September 2010 Published Online First 19 November 2010

\title{
Long-term results of autologous cultivated oral mucosal epithelial transplantation in the scar phase of severe ocular surface disorders
}

\author{
Takahiro Nakamura, ${ }^{1,2}$ Kazunori Takeda, ${ }^{1}$ Tsutomu Inatomi, ${ }^{1}$ Chie Sotozono, ${ }^{1}$ \\ Shigeru Kinoshita ${ }^{1}$
}

\section{ABSTRACT}

Purpose To investigate the long-term outcome of autologous cultivated oral mucosal epithelial transplantation (COMET) for the treatment of the scar phase of severe ocular surface disorders.

Participants This study involved 19 eyes of 17 patients who received autologous COMET for total limbal stem-cell deficiency.

Methods Autologous cultivated oral mucosal epithelial sheets were created using amniotic membrane as a substrate. Clinical efficacy was evaluated by best-corrected visual acuity and visual acuity at the postoperative 36th month. The clinical results (clinical conjunctivalisation, corneal opacification, corneal neovascularisation and symblepharon formation) were evaluated and graded on a scale from 0 to 3 according to their severity. Clinical safety was evaluated by the presence of persistent epithelial defects, ocular hypertension and infections.

Results Autologous cultivated oral mucosal epithelial sheets were successfully generated for all 17 patients. All patients were followed up for more than 36 months; the mean follow-up period was 55 months and the longest follow-up period was 90 months. During the long-term follow-up period, postoperative conjunctivalisation and symblepharon were significantly inhibited. All eyes manifested various degrees of postoperative corneal neovascularisation, but it gradually abated and its activity was stable at 6 months after surgery. Best-corrected visual acuity was improved in 18 eyes (95\%) during the follow-up periods, and visual acuity at the postoperative 36th month was improved in 10 eyes (53\%).

Conclusions These long-term clinical results strongly support the conclusion that tissue-engineered cultivated oral mucosal epithelial sheets are useful in reconstructing the ocular surface of the scar phase of severe ocular surface disorders.

\section{INTRODUCTION}

Severe ocular surface disease (OSD), such as Stevens-Johnson syndrome (SJS) and ocular cicatricial pemphigoid (OCP), can be devastating and result in significant visual complications. ${ }^{1-5}$ Since 1997, with the knowledge of tissue-specific stem cell behaviour and the development of tissue-engineering techniques, cultivated corneal limbal epithelial transplantation (CLET) has been shown to be a promising treatment modality for the management of severe OSD. ${ }^{6-12}$ However, as most severe OSD is bilateral, surgeons were forced to use allograft donor cells, which subject the recipients to a high risk for allogeneic rejection and necessitate prolonged immunosuppression therapy. More recently, our experimental ${ }^{13}$ and serial clinical studies $^{14-18}$ demonstrated the efficacy of autologous cultivated oral mucosal epithelial transplantation (COMET) for the treatment of severe OSD. Even though initial clinical results of COMET have been reported from several groups worldwide, ${ }^{19} 20$ including our group, the long-term clinical assessments of COMET are entirely unknown and the feasibility of this technique still requires detailed investigation.

Here, we present the long-term clinical data on 19 eyes that received COMET, for which the mean follow-up period was 55 months; the longest follow-up period being 90 months. This study has important clinical implications and provides new information regarding the long-term visual results and survival of transplanted cultivated cells for the treatment of the scar phase of severe OSD cases.

\section{MATERIALS AND METHODS Subjects}

All experimental procedures and clinical applications introduced in this study were approved by the Institutional Review Board for Human Studies of Kyoto Prefectural University of Medicine; prior informed consent was obtained from all patients in accordance with the tenets of the Declaration of Helsinki for research involving human subjects.

The study included 19 eyes from 17 patients with the scar phase of severe OSD who underwent ocular surface reconstruction with COMET at our hospital from August 2002 to January 2007, and who could be followed up for more than 36 months. In this study, to precisely examine the long-term clinical results of COMET for corneal surface reconstruction, we excluded the patients who received penetrating keratoplasty after the initial COMET and patients who received COMET for conjunctival fornix reconstruction. The patients included 7 males and 10 females; their ages ranged from 20 to 80 years (mean age: $54 \pm 21$ years). The patients were followed up for a mean period of $55 \pm 17$ months; the longest follow-up period was 90 months. All patients were diagnosed as totally stem-cell-deficient on the basis of complete disappearance of the palisades of Vogt and $360^{\circ}$ of conjunctivalisation. The preoperative diagnosis was SJS in 11 eyes, OCP in 4 eyes, squamous cell carcinoma in 2 eyes, thermal or chemical injury in 1 eye and graft-versus-host disease in 1 eye. Tear production was diminished but not absent in all 
patients, as evidenced by the presence of a tear meniscus level with diminished tear-film break-up time.

\section{Cultivation of oral mucosal epithelial sheets}

We cultured human oral mucosal epithelial cells using a previously reported system. ${ }^{14-18}$ Briefly, the presence of healthy oral mucosa was first confirmed by a dentist before biopsy. A small oral mucosal biopsy was performed under local anaesthesia. The oral epithelium was then incubated at $4^{\circ} \mathrm{C}$ for $5 \mathrm{~h}$ with $1.2 \mathrm{IU}$ Dispase, followed by treatment with $0.05 \%$ Trypsin-EDTA solution for $10 \mathrm{~min}$ to separate the cells. The resultant oral epithelial cells $\left(1-2 \times 10^{5} \mathrm{cell} / \mathrm{ml}\right)$ were then seeded onto denuded amniotic membrane (AM) spread on the bottom of culture inserts and co-cultured with mitomycin C (MMC)inactivated 3T3 fibroblasts. The culture medium consisted of defined keratinocyte growth medium (KGM: ArBlast Co., Ltd., Kobe, Japan) supplemented with $5 \%$ serum. The cultured cells were submerged in medium for 2 weeks and then air-lifted for 1-2 days by lowering the medium level.

\section{Surgical procedure}

The surgical procedure was as described in our previous report. ${ }^{14-18}$ Briefly, we performed a $360^{\circ}$ conjunctival peritomy $3 \mathrm{~mm}$ from the limbus and removed all perilimbal scarred or inflamed subconjunctival tissue down to bare sclera. The corneal pannus was completely removed by blunt dissection or superficial keratectomy using surgical scissors or a blade. The cultivated oral mucosal epithelial sheet was placed over the corneal surface and secured in place with 10-0 nylon sutures at the limbus. The integrity of the cultivated epithelium was confirmed by fluorescein staining at the end of the surgery, and the ocular surface was protected with a medical-use bandage contact lens (Plano B4). Postoperatively, three types of medicaluse bandage contact lenses (Plano B4, Acuvue and $\mathrm{O}_{2}$ Optics) were properly used within 1-3 months after surgery depending on the condition of the corneal surface.

Postoperatively, $0.3 \%$ ofloxacin and $0.1 \%$ dexamethasone eye drops were instilled four times a day. The doses were tapered to a maintenance dose of - two to three times a day after 2-3 months, depending on the severity of inflammation. Oral betamethasone (1 mg/day) and cyclosporine (100 mg/day) were administered to reduce inflammation and were tapered down and then stopped 1 month postoperatively.

\section{Evaluation of clinical efficacy}

Preoperative visual acuity (VA), postoperative best-corrected visual acuity (BCVA) and VA at the postoperative 36th month were measured, and the ocular surface was inspected with a slitlamp microscope and fluorescence staining. The clinical results (clinical conjunctivalisation (eg, invasion of conjunctival tissue), corneal opacification, corneal neovascularisation and symblepharon formation) were evaluated by two ophthalmologists and graded on a scale from 0 to 3 according to their severity in accordance with our previously reported grading system. ${ }^{21}$

\section{Evaluation of clinical safety}

For the assessment of postoperative complications, the patient's eyes were carefully examined for persistent epithelial defects (PEDs), ocular hypertension and infections. Epithelial defects were considered persistent if they lasted for more than 4 weeks. Ocular hypertension was considered a postoperative complication if it had not been present preoperatively. When we clearly observed the clinical bacterial focus region in the cornea, we considered it a corneal infection.

\section{RESULTS}

There were no complications during or after the excision of the oral mucosa. Autologous cultivated oral mucosal epithelial sheets were successfully generated for all 17 patients, but one case was merely fair because only $70 \%$ of the entire cultivated epithelial sheet showed mature stratification as determined by fluorescein staining under a phase-contrast microscope and an operating microscope at the end of surgery. All eyes, including the eye transplanted with the sheet whose quality we judged as only fair by use of an inverted microscope, demonstrated total re-epithelialisation of the corneal surface within 2-7 days after surgery. Successful engraftment was initially achieved in all patients with none of the grafts sloughing off. Combined surgery was performed as follows: subconjunctival MMC treatment (17 eyes, 90\%), amniotic membrane transplantation (AMT) (14 eyes, 74\%) and cataract surgery (5 eyes, 26\%).

Preoperative BCVA in our series was light perception, hand motion (HM) or finger counting (15 eyes, 79\%) and worse than 20/500 (4 eyes, 21\%). Postoperative VA (BCVA, and VA at the postoperative 36 th month) is summarised in figure 1 and table 1. Postoperative visual recovery ranged from HM to 20/40; during the follow-up, BCVA was improved more than 2 lines in 15 eyes $(79 \%)$ and VA at the postoperative 36th month was improved in 8 eyes (42\%).

The clinical grading scores pertaining to conjunctivalisation, corneal opacity, corneal neovascularisation and symblepharon formation were evaluated, and it was found that during the longterm follow-up period, postoperative clinical conjunctivalisation
Figure 1 Postoperative best-corrected visual acuity (BCVA) (A) and visual acuity (VA) at the postoperative 36th month (B). The diagonal line indicates the values at which the preoperative and postoperative values for visual acuity were the same. CF: counting fingers; HM: hand motion; LP: light perception.
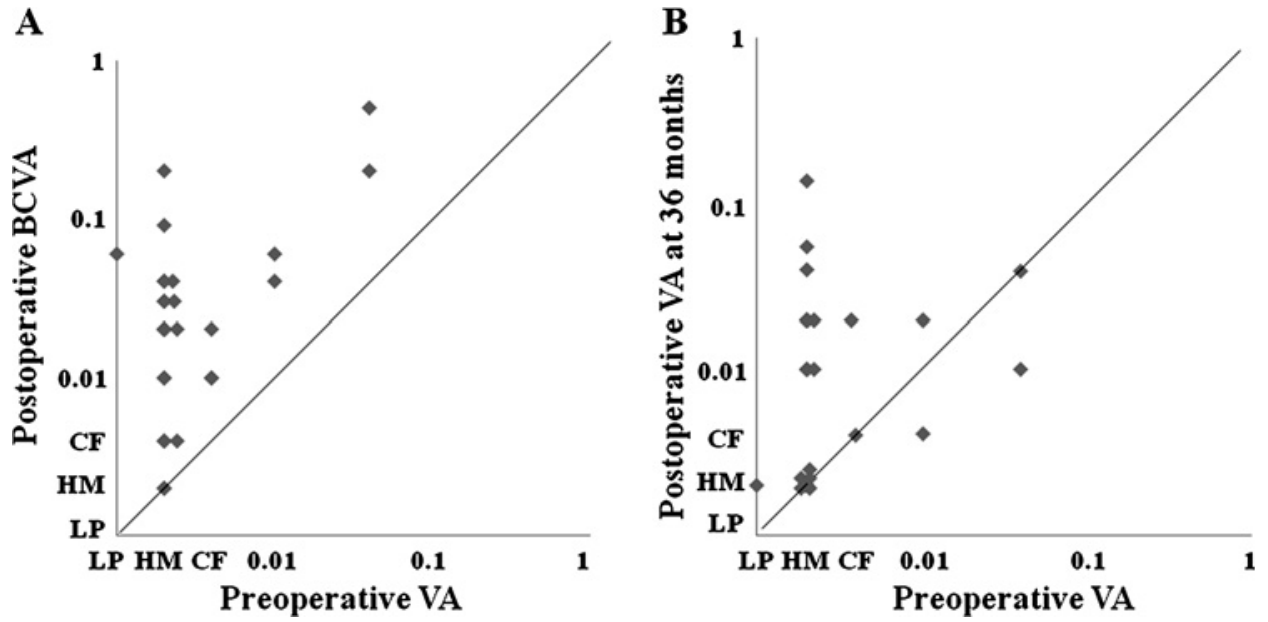
Table 1 Summary of postoperative VA

\begin{tabular}{lll}
\hline & $\begin{array}{l}\text { Postoperative } \\
\text { BCVA (\%) }\end{array}$ & $\begin{array}{l}\text { Postoperative VA } \\
\text { at } 36 \text { months (\%) }\end{array}$ \\
\hline Improvement of VA (more than two lines) & 79 & 42 \\
Improvement of VA (more than one line) & 95 & 53 \\
No change & 5 & 37 \\
Decline & 0 & 11 \\
\hline
\end{tabular}

BCVA, best-corrected visual acuity; VA, visual acuity.

and symblepharon were significantly inhibited (figure 2). Corneal opacification tended to improve. All eyes manifested various degrees of superficial corneal vascularisation, but it gradually abated and its activity was comparatively stable from 6 months after surgery. We theorise that postoperative neovascularisation occurs because in vivo oral mucosa requires a vascular bed for maintenance.

Regarding the postoperative complications, a small but persistent epithelial defect was observed in 5-26\% of the patients during the follow-up periods (table 2). Of the total 19 eyes, 7 eyes (37\%) manifested PEDs once during the follow-up periods, while postoperative ocular hypertension was observed in 3 eyes (16\%) (table 2). Although the intraocular pressure (IOP) of those three patients was occasionally high, they did not require glaucoma surgery. The occasional increase in IOP was mainly managed by the administration of carbonic anhydrase inhibitor (two eyes), or by the topical application of $0.05 \%$ latanoprost (one eye) or carteolol hydrochloride (one eye). Methicillin-resistant Staphylococcus aureus (MRSA) was the only cause of postoperative corneal infection (one eye), and that corneal infection was observed only within 6 months after transplantation (table 2).

The clinical progress of three representative patients with total limbal stem cell deficiency arising from SJS and idiopathic OSD is shown in figure 3. Before transplantation all eyes manifested severe destruction of the ocular surface with limbal stem cell deficiency (figure 3A,C,E). Postoperative appearance at 50 (figure $3 \mathrm{~B}$ ) and 71 (figure $3 \mathrm{D}$ ) months shows a relatively smooth, epithelialised corneal surface with minimal corneal neovascularisation, scarring and inflammation. Postoperative appearance at 72 months (figure 3F) shows that due to the PED during the follow-up periods, postoperative corneal opacity was stronger than in other cases with modest neovascularisation, finally affecting the transparency of the cornea even though the ocular surface was relatively stable.

\section{DISCUSSION}

Ocular surface reconstruction for severe OSD continues to be one of the most challenging fields in ophthalmology. COMET is the most recent therapeutic method for the treatment of severe OSD, and this study provides new information regarding the long-term results of this new treatment. We found through our earlier preliminary and mid-term clinical results ${ }^{14-18}$ that COMET is an efficacious treatment for severe OSD. The overall success rate, as measured by the improvement of BCVA and VA at the postoperative 36 th month, was $95 \%$ and $53 \%$, respectively. This success rate is similar to that of a previous report, and the patients who participated in this study were some of the most severe cases with their preoperative VA all being worse than 20/500, suggesting that our clinical results were fair and that COMET was useful for reconstructing the ocular surface of these patients with severe OSD.

In 1999, our group started the clinical application of allogeneic CLET and since 2002 we have been performing COMET for patients with severe OSD. Through these many years of clinical experience we have learned a great deal from our clinical and biological findings as follows: (1) Wearing a therapeutic soft contact lens in the early postoperative period is essential for the clinical success of COMET because it provides protection for the epithelial cells from mechanical ablation; we first used the relatively rigid-type soft contact lens (eg, the Plano B4), which is
Figure 2 The results of the postoperative observation time course of clinical grading score regarding conjunctivalisation $(A)$, corneal opacity (B), corneal neovascularisation (C), and symblepharon formation (D).
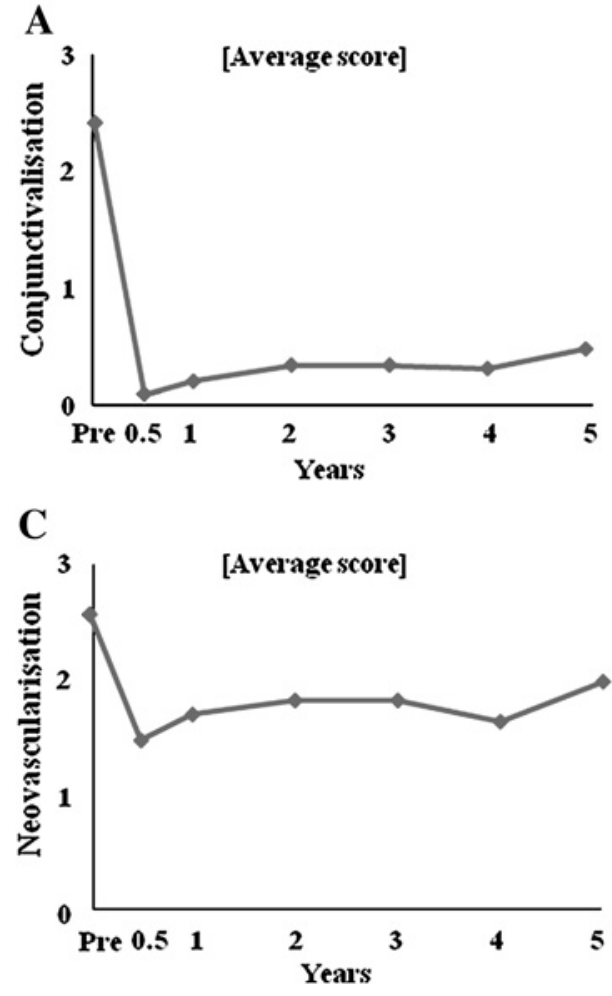

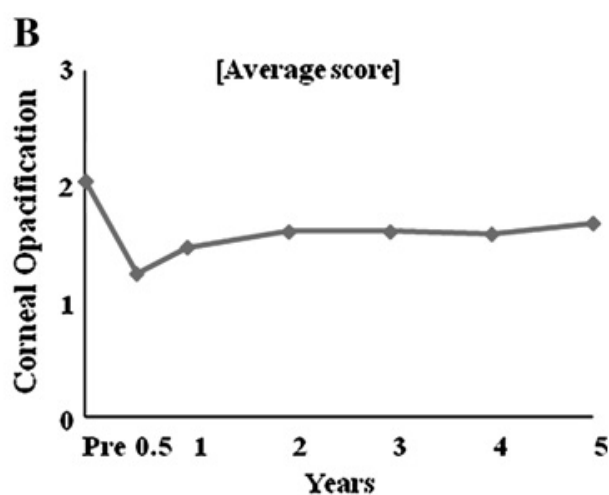

D

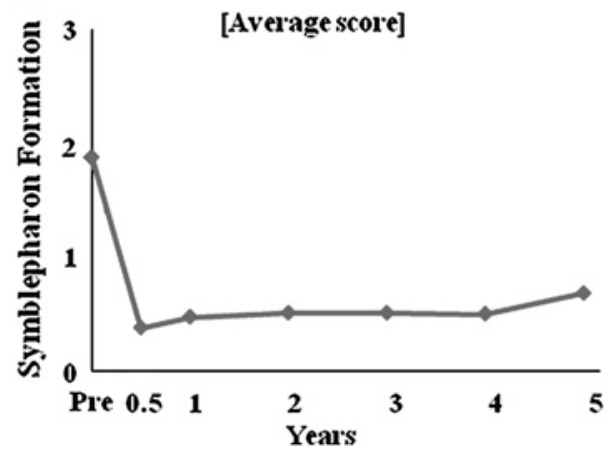


Table 2 Time course of postoperative complications

\begin{tabular}{llllllll}
\hline & Pre & $\mathbf{6 M}$ & $\mathbf{1 2 M}$ & $\mathbf{2 4 M}$ & $\mathbf{3 6 M}$ & $\mathbf{4 8 M}$ & $\mathbf{6 0 M}$ \\
\hline Persistent epithelial defect & $5 \%$ & $26 \%$ & $5 \%$ & $5 \%$ & $5 \%$ & $8 \%$ & $0 \%$ \\
& $(1 / 19)$ & $(5 / 19)$ & $(1 / 19)$ & $(1 / 19)$ & $(1 / 19)$ & $(1 / 12)$ & $(0 / 8)$ \\
Ocular hypertension & $5 \%$ & $10 \%$ & $15 \%$ & $5 \%$ & $5 \%$ & $8 \%$ & $12 \%$ \\
\multirow{4}{*}{ Infection } & $(1 / 19)$ & $(2 / 19)$ & $(3 / 19)$ & $(1 / 19)$ & $(1 / 19)$ & $(1 / 12)$ & $(1 / 8)$ \\
& $0 \%$ & $5 \%$ & $0 \%$ & $0 \%$ & $0 \%$ & $0 \%$ & $0 \%$ \\
& $(0 / 19)$ & $(1 / 19)$ & $(0 / 19)$ & $(0 / 19)$ & $(0 / 19)$ & $(0 / 12)$ & $(0 / 8)$ \\
\hline
\end{tabular}

M, months.

difficult to take off, and then we used the highly hydrated-type soft contact lens (Acuvue, $\mathrm{O}_{2}$ Optics) to improve the permeability of oxygen to the corneal surface and to prevent epithelial damage due to lack of oxygen. (2) Within 1 week of COMET, almost all patients encountered dry-eye conditions, the severity of which depended upon the individual patient. Therefore, artificial eye drops should be instilled frequently (from every 15 min to $2 \mathrm{~h}$ ).

Even though the morphological appearance of a cultivated oral mucosal epithelial sheet is quite similar to that of a cultivated corneal limbal epithelial sheet, one of the most distinguishable characteristics of a cultivated oral mucosal epithelial sheet is its distinctive fluorescein staining pattern. From our clinical experience, its staining pattern is more like that of superficial punctate keratopathy than conjunctival epithelium, but a strict discrimination between the two is somewhat difficult to observe by slit-lamp examination. Although the transplanted cultivated oral mucosal epithelial sheets in this study retained their transparency, there was a slightly high amount of light-scattering under the slit-lamp microscope examination, with or without fluorescein staining, thus affecting the postoperative corneal opacity and resulting in a BCVA of 20/40 in the 19 eyes treated by COMET. For most eyes, the BCVA was between 20/2000 and 20/200, suggesting that light-scattering on the transplanted oral mucosal epithelium on the cornea reached the level that controls VA at around 20/500. In contrast, the BCVA of some CLET patients reached 20/20, indicating that the biological character of the cells clearly affects the quality of VA.
Figure 3 The clinical progress of three representative patients with severe OSD arising from SJS $(A, B)$, idiopathic OSD $(C, D)$ and SJS (E, F). Before transplantation all eyes manifested severe destruction of the ocular surface with limbal stem cell deficiency ( $A, C$ and $\mathrm{E})$. The postoperative appearance at 50 (B) and 71 (D) months shows a relatively smooth, epithelialised corneal surface with minimal corneal neovascularisation, scarring and inflammation. The postoperative appearance at 72 months (F) shows that due to the persistent epithelial defect during the follow-up periods, postoperative corneal opacity is somehow remarkable with modest neovascularisation, finally affecting the transparency of the cornea even though the ocular surface is relatively stable. OSD, ocular surface disease; SJS, Stevens-Johnson syndrome.
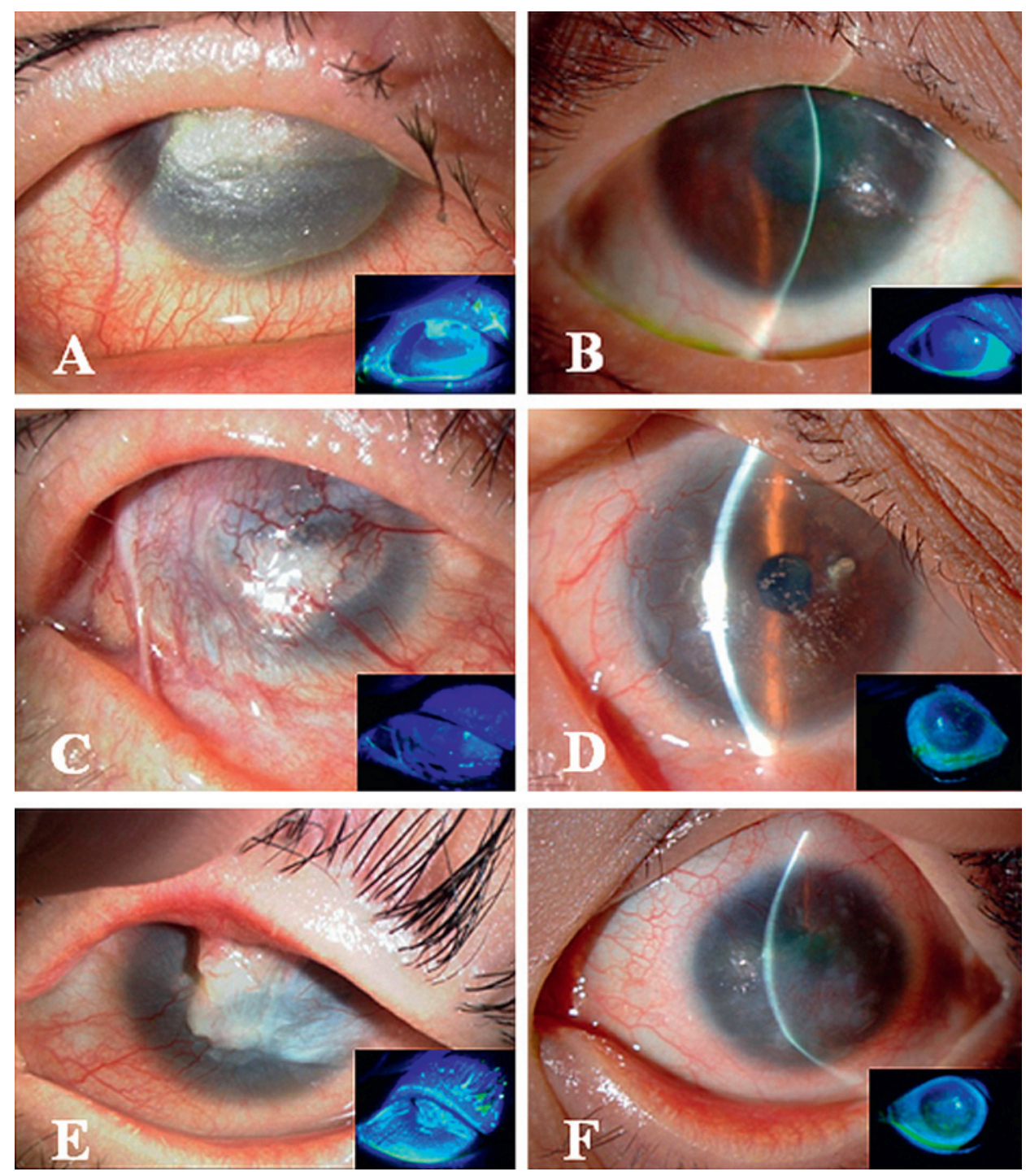
The patients with OSD reported here were the most severe cases we encountered, necessitating the reconstruction of not only the corneal surface but also the surrounding ocular surface. Of the 19 eyes in this study, $90 \%$ and $74 \%$ of the cases received treatment by MMC and AMT, respectively, to prevent the postoperative proliferation activity of the subconjunctival tissue and to reconstruct the ocular surface including the conjunctival fornix. After COMET, transplanted cultivated oral epithelial cells were always observed to migrate outwards on the AM, ultimately covering the complete ocular surface. Through the simultaneous combination of these procedures, postoperative symblepharon formation was significantly inhibited during the long-term follow-up and we believe that this is the one of the most beneficial advantages of COMET. In addition, of the 19 eyes, $26 \%$ of the cases simultaneously received cataract surgery to improve the VA. Even though the intraocular visibilities in patients with OSD were often very bad, we developed the surgical technique as a step-by-step process and can now perform it safely through the use of a surgical slit-lamp microscope and indocyanine green staining of the anterior lens capsule.

We carefully assessed the clinical safety of COMET and found that postoperative PED sometimes occurred in our series. Of the 19 eyes, PED occurred in 7 eyes (37\%) at least once during the long-term follow-up. Of those seven eyes, five eyes (71\%; all with SJS) were systemic primary OSD. It has been reported that the health of the oral mucosal epithelium in vivo depends on the existing diseases. ${ }^{22}$ Even though we were able to generate a cultivated oral mucosal epithelial sheet from systemic primary OSD, whose morphological features are quite similar to in vivo corneal epithelium, the biological ability of the oral epithelium cells may potentially be damaged in these patients. This issue is currently under investigation in our laboratory.

Of the 19 eyes, ocular hypertension was observed in a total of 3 eyes $(16 \%)$ during the postoperative follow-up period. Since the transplanted cultivated sheets were not completely identical to in vivo epithelium, care must be taken in regard to postoperative epithelial damage caused by the use of antiseptic eye drops. Thus, a major clinical point is that in two eyes in this study, the occasional increase in IOP was found to be better managed by the administration of a carbonic anhydrase inhibitor.

In our series, postoperative corneal infections were relatively few (one eye, 5\%) as compared to allogeneic CLET or limbal transplantation, ${ }^{9} 23$ simply because COMET is an autologous transplantation and patients did not need the intensive, prolonged postoperative immunosuppressant therapy, thus resulting in the avoidance of an immunocompromised state. Interestingly, in view of the findings in this study and our recent clinical experiences, the postoperative corneal infection in our cases mainly occurred within 6 months after surgery, and all of those cases were systemic OSD patients. Furthermore, all of those cases were detected to be caused by MRSA. ${ }^{24}$ It has been reported that MRSA is frequently detected from the ocular surface in patients with SJS as compared with normal subjects; therefore, it is important to carefully observe the corneal surface in the early postoperative periods, especially in patients with SJS.

In conclusion, this is the first study that demonstrates the long-term clinical results of COMET for ocular surface reconstruction in the treatment of the scar phase of severe OSD. We found that COMET permits sustained reconstruction of the ocular surface epithelium in many eyes with severe OSD. The management of postoperative PED and neovascularisation may further increase the efficacy of this type of transplantation.
Acknowledgements We thank Mr J Bush for editing our manuscript, and Ms Saito, Horikiri and Mano for assisting with the culture procedures.

Funding Supported in part by Grants-in-Aid for scientific research from the Japanese Ministry of Health, Labour and Welfare (H16-Saisei-007), and the Japanese Ministry of Education, Culture, Sports, Science and Technology (Kobe Translational Research Cluster), a research grant from the Kyoto Foundation for the Promotion of Medical Science, and the Intramural Research Fund of Kyoto Prefectural University of Medicine.

\section{Competing interests None.}

Ethics approval This study was conducted with the approval of the Institutional Review Board for Human Studies of Kyoto Prefectural University of Medicine.

Provenance and peer review Not commissioned; externally peer reviewed.

\section{REFERENCES}

1. Shapiro MS, Friend J, Thoft RA. Corneal re-epithelialization from the conjunctiva. Invest Ophthalmol Vis Sci 1981;21:135-42.

2. Dua H, Forrester JV. The corneoscleral limbus in human corneal epithelial wound healing. Am J Ophthalmol 1990;110:646-56.

3. Tsai RJF, Sun TT, Tseng SC. Comparison of limbal and conjunctival autograft transplantation in corneal surface reconstruction in rabbits. Ophthalmology 1990;97:446-55

4. Thoft RA. Keratoepithelioplasty. Am J Ophthalmol 1984;97:1-6.

5. Kenyon KR, Tseng SC. Limbal autograft transplantation for ocular surface disorders. Ophthalmology 1989;96:709-23.

6. Pellegrini G, Traverso CE, Franzi AT, et al. Long-term restoration of damaged corneal surfaces with autologous cultivated corneal epithelium. Lancet 1997;349:990-3.

7. Tsai RJ, Li LM, Chen JK. Reconstruction of damaged corneas by transplantation of autologous limbal epithelial cells. N Engl J Med 2000;343:86-93.

8. Schwab IR, Reyes M, Isseroff RR. Successful transplantation of bioengineered tissue replacements in patients with ocular surface disease. Cornea 2000;19: 421-6.

9. Koizumi N, Inatomi T, Suzuki T, et al. Cultivated corneal epithelial stem cell transplantation in ocular surface disorders. Ophthalmology 2001;108:1569-74.

10. Rama $\mathbf{P}$, Bonini $S$, Lambiase $A$, et al. Autologous fibrin-cultured limbal stem cells permanently restore the corneal surface of patients with total limbal stem cell deficiency. Transplantation 2001;72:1478-85.

11. Grueterich $\mathbf{M}$, Espana EM, Touhami A, et al. Phenotypic study of a case with successful transplantation of ex vivo expanded human limbal epithelium for unilateral total limbal stem cell deficiency. Ophthalmology 2002;109:1547-52.

12. Shimazaki J, Aiba M, Goto E, et al. Transplantation of human limbal epithelium cultivated on amniotic membrane for the treatment of severe ocular surface disorders. Ophthalmology 2002;109:1285-90.

13. Nakamura T, Endo K, Cooper LJ, et al. The successful culture and autologous transplantation of rabbit oral mucosal epithelial cells on amniotic membrane. Invest Ophthalmol Vis Sci 2003:44:106-16.

14. Nakamura T, Inatomi T, Sotozono C, et al. Transplantation of cultivated autologous oral mucosal epithelial cells in patients with severe ocular surface disorders. $\mathrm{Br} \mathrm{J}$ Ophthalmol 2004;88:1280-4.

15. Inatomi T, Nakamura T, Koizumi N, et al. The mid-term results of ocular surface reconstruction using cultivated autologous oral mucosal epithelial transplantation. Am J Ophthalmol 2006;141:267-75.

16. Inatomi T, Nakamura T, Kojyo $\mathrm{M}$, et al. Ocular surface reconstruction with combination of cultivated autologous oral mucosal epithelial transplantation and penetrating keratoplasty. Am J Ophthalmol 2006;142:757-64.

17. Ang LPK, Nakamura T, Inatomi T, et al. Autologous serum-derived cultivated oral epithelial transplants for severe ocular surface disease. Arch Ophthalmol 2006;124:1543-51

18. Nakamura T, Inatomi T, Cooper LJ, et al. Phenotypic investigation of human eyes with transplanted autologous cultivated oral mucosal epithelial sheets for severe ocular surface diseases. Ophthalmology 2007;114:1080-8.

19. Nishida K, Yamamoto $\mathrm{M}$, Hayashida $\mathrm{Y}$, et al. Corneal reconstruction with tissue-engineered cell sheets composed of autologous oral mucosal epithelium. N Engl J Med 2004;351:1187-96.

20. Satake Y, Dogru M, Yamane GY, et al. Barrier function and cytologic features of the ocular surface epithelium after autologous cultivated oral mucosal epithelial transplantation. Arch Ophthalmol 2008;126:23-8.

21. Sotozono C, Ang LPK, Koizumi N, et al. New grading system for the evaluation of chronic ocular manifestations in patients with Stevens-Johnson syndrome. Ophthalmology 2007;114:1294-302.

22. Scully C, Bagan J. Oral mucosal diseases: erythema multiforme. Br J Oral Maxillofac Surg 2008;46:90-5.

23. Tsubota K, Satake $Y$, Kaido M, et al. Treatment of severe ocular-surface disorders with corneal epithelial stem-cell transplantation. N Engl J Med 1999;340 1697-703.

24. Sotozono C, Inagaki K, Fujita A, et al. Methicillin-resistant Staphylococcus aureus and methicillin-resistant Staphylococcus epidermidis infections in the cornea. Cornea 2002;21(7 Suppl):S94-101. 